\title{
Caractéristiques de l'environnement bâti du quartier associées à différents types d'activité physique chez les adultes canadiens
}

\author{
Gavin R. McCormack, Ph. D.
}

Cet article a fait l'objet d'une évaluation par les pairs.

\section{Résumé}

Introduction : L'étude tente d'estimer les associations entre les caractéristiques du milieu bâti d'un quartier et la marche comme moyen de transport (MT), la marche récréative (MR), l'activité physique à intensité modérée (APM) et l'activité physique à intensité élevée (APE) chez les adultes, indépendamment des caractéristiques sociodémographiques et de l'autosélection du lieu de résidence (les raisons liées à l'activité physique associées au choix d'un quartier par quelqu'un).

\begin{abstract}
Méthodologie : En 2007 et 2008, 4423 adultes de Calgary ont répondu à des entrevues au téléphone fixe portant sur l'activité physique, leurs caractéristiques sociodémographiques et les raisons de l'autosélection de leur résidence. À l'aide de données spatiales, nous avons estimé la densité de population, la proportion d'espaces verts, la longueur des sentiers et des pistes cyclables, la densité commerciale, la densité des arrêts d'autobus, la densité des arbres gérés par la ville, la longueur de trottoir, les divers types de parcs et l'offre de destinations récréatives dans un rayon de 1,6 km en fonction du code postal résidentiel géolocalisé des participants. Des modèles linéaires généralisés ont été utilisés pour estimer les associations entre les caractéristiques de l'environnement bâti du quartier et la participation à des activités physiques hebdomadaires dans le quartier (10 minutes/ semaine ou plus, rapports de cotes $[\mathrm{RC}]$ ) et, parmi les personnes ayant déclaré une participation, la durée de l'activité (coefficients bêta [B] non normalisés).
\end{abstract}

Résultats : L'échantillon comprenait davantage de femmes (59,7 \%) que d'hommes (40,3\%) et l'âge moyen (écart-type) était de 47,1 ans (15,6 ans). L'utilisation de la MT était associée à la densité des intersections ( $\mathrm{RC}=1,11$; IC à $95 \%: 1,03$ à 1,20) et des commerces $(\mathrm{RC}=1,52 ; 129$ à 1,78$)$ et à la longueur des trottoirs $(\mathrm{RC}=1,19 ; 1,09$ à $1,29)$ alors que le nombre de minutes de MT était associé à la densité commerciale $(\mathrm{B}=19,24$ minutes/semaine; 11,28 à 27,20), à la densité d'arbres $(\mathrm{B}=6,51 ; 2,29$ à 10,72 minutes/semaine) et à l'offre de destinations récréatives ( $\mathrm{B}=-8,88$ minutes/ semaine, $-12,49$ à $-5,28)$. L'utilisation de la MR était associée à la longueur des sentiers ou pistes cyclables $(\mathrm{RC}=1,17 ; 1,05$ à 1,31). Avoir une APM était associé à l'offre de destinations récréatives ( $\mathrm{RC}=1,09 ; 1,01$ à 1,17 ) et à la longueur de trottoir $(\mathrm{RC}=1,10 ; 1,02$ à 1,19$)$, alors que le nombre de minutes d'APM était associé négativement à la densité de population ( $\mathrm{B}=-8,65$ minutes/semaine; $-15,32$ à $-1,98)$. Avoir une APE était associé à la longueur de trottoir $(\mathrm{RC}=1,11 ; 1,02$ à 1,20$)$, à la longueur des sentiers ou pistes cyclables $(\mathrm{RC}=1,12 ; 1,02$ à 1,24$)$ et à la proportion d'espaces verts dans le quartier ( $\mathrm{RC}=0,89 ; 0,82$ à 0,98$)$. Le nombre de minutes d'APE était associé à la densité d'arbres ( $\mathrm{B}=7,28$ minutes/semaine; 0,39 à 14,17).

Conclusion : Certaines caractéristiques de l'environnement bâti du quartier semblent importantes pour favoriser la pratique d'activité physique, tandis que d'autres peuvent être plus propices à l'augmentation de la durée de l'activité physique. Les modifications augmentant la densité de destinations utilitaires et la quantité de trottoirs dans les quartiers seraient susceptibles d'augmenter les niveaux totaux d'activité physique dans les quartiers.

Mots-clés : activité physique, accessibilité piétonnière, quartier, forme urbaine, environnement bâti
Diffuser cet article sur Twitter

Points saillants

- Les caractéristiques de l'environnement bâti des quartiers sont importantes pour favoriser, outre la marche, différents types d'activité physique effectuée dans le quartier.

- Les caractéristiques de l'environnement bâti, en particulier les trottoirs et les destinations non récréatives dans un rayon de moins de 1,6 km $\mathrm{du}$ domicile, pourraient favoriser chez les adultes une augmentation des taux globaux d'activité physique effectuée dans le quartier.

- Les politiques qui favorisent la création de milieux bâtis propices à l'activité physique dans les villes canadiennes pourraient contribuer à l'augmentation de l'activité physique et, par conséquent, à l'amélioration de la santé de la population.

\section{Introduction}

Moins d'un adulte canadien sur cinq atteint les niveaux d'activité physique jugés nécessaires à une santé optimale ${ }^{1,2}$. En outre, les adultes canadiens consacrent en moyenne seulement trois minutes par jour environ à des activités physiques d'intensité élevée (c.-à-d. une activité physique qui demande une dépense d'énergie au moins six fois plus élevée que celle associée au repos) ${ }^{2}$. Les adultes canadiens consacrent en moyenne beaucoup plus de temps (par rapport à une activité physique intense), soit environ 20 minutes par jour, à des activités physiques d'intensité modérée (qui demandent une dépense d'énergie de trois à cinq fois plus grande que celle associée au repos) ${ }^{2}$. L'activité physique de forte intensité est cependant susceptible de procurer des bienfaits pour la santé bien supérieurs

Rattachement de l'auteur :

Département des sciences de la santé communautaire, Université de Calgary, Calgary (Alberta), Canada

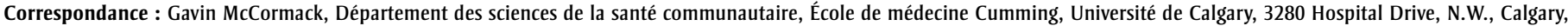
AB T2N 4Z6; tél. : 403-220-8193; téléc. : 403-210-3818; courriel : gmccorma@ucalgary.ca 
à ceux généralement associés à une activité physique d'intensité modérée ${ }^{3-7}$. Une fois les données ajustées en fonction de la dépense énergétique totale, on constate que la pratique d'une activité physique d'intensité élevée offre des avantages cardiovasculaires plus importants que celle d'une activité physique d'intensité modérée ${ }^{7}$. L'activité physique d'intensité élevée est associée de façon positive à une meilleure condition physique aérobie ${ }^{7,8}$ et de façon négative au risque de maladie chronique et de mortalité toutes causes confondues $^{9}$. Ainsi, l'augmentation des niveaux d'activité physique tant modérée qu'élevée est importante pour réduire les problèmes de santé chroniques comme les maladies cardiovasculaires, le diabète de type 2, l'hypertension, la dépression, le surpoids et l'obésité ainsi que certains cancers, qui imposent un fardeau important au système de soins de santé canadien ${ }^{10}$.

Des facteurs intraindividuels, interindividuels et environnementaux spécifiques pourraient avoir une influence sur le type et l'intensité de l'activité physique à laquelle s'adonnent les adultes ${ }^{11}$. Malgré l'émergence depuis 20 ans de données sur les liens entre environnement bâti et activité physique, la plupart des études les ayant produites portent seulement sur les relations entre les caractéristiques de l'environnement bâti du quartier et la marche et le vélo ${ }^{12,13}$. On observe une corrélation constante entre les caractéristiques de l'environnement bâti (diverses formes d'occupation des sols, densité résidentielle, liens piétonniers et accessibilité piétonnière en général) et la marche ${ }^{12}$. Et, même si certaines caractéristiques de l'environnement bâti sont associées aux deux types de marche, il semble que certaines d'entre elles favorisent davantage la marche comme moyen de transport et d'autres, la marche récréative $e^{12,14,15}$. De même, les chercheurs ont découvert que différentes caractéristiques de l'environnement bâti ont une influence sur l'intensité de l'activité physique (c.-à-d. marche, activité physique d'intensité modérée ou activité physique d'intensité élevée) ${ }^{8,13,16}$. Même si les études sur les relations entre environnement bâti et activité physique à forte intensité sont rares, les résultats suggèrent que les caractéristiques de l'environnement construit du quartier, autodéclarées aussi bien mesurées objectivement, comme les trottoirs ${ }^{17}$, les pistes cyclables ${ }^{8}$, les espaces verts et les espaces ouverts de qualité ${ }^{8}$, la présence de monuments ${ }^{18}$, la densité des intersections ${ }^{19}$, la densité des voies locales ${ }^{19}$, la proximité, la disponibilité et l'utilisation des installations liées à l'activité physique ${ }^{16,17,20-22}$, la sécurité $^{17}$, l'esthétique et les points de vue intéressants ${ }^{8,17}$ ainsi que la facilité de marche $^{23}$ jouent potentiellement un rôle important pour faciliter l'activité physique de forte intensité. Le fait de mieux connaître les caractéristiques propres à l'environnement bâti associées à chaque type d'activité physique pourrait contribuer à une planification et à un aménagement de quartiers favorables à la santé13,24.

L'autosélection du lieu de résidence, à savoir le processus non aléatoire qui préside au choix d'un quartier correspondant aux préférences d'une personne en matière d'activité physique, complique le travail des chercheurs s'intéressant au lien entre environnement bâti et activité physique, en particulier parce qu'il affecte les données probantes dérivées des méthodes d'études transversales. Si elle n'est pas statistiquement contrôlée ou ajustée dans les études transversales, l'autosélection résidentielle peut conduire à une surestimation de l'association entre les caractéristiques du milieu bâti et l'activité physique ${ }^{14,25}$. Seules quelques études transversales ont estimé les associations entre l'environnement bâti et l'activité physique en tenant compte sur le plan statistique de l'autosélection résidentielle $^{14}$. Les données autodéclarées sur l'activité physique par quartier sont par ailleurs rarement mesurées. Les mesures de l'activité physique qui ignorent le contexte dans lequel le comportement est effectué (par exemple dans le quartier) peuvent sous-estimer les associations véritables entre les caractéristiques de l'environnement bâti et l'activité physique ${ }^{24}$. Le fait de tenir compte de l'autosélection résidentielle, de noter les activités physiques habituellement pratiquées l'été et l'hiver et de recueillir des données sur l'activité physique spécifique au quartier est susceptible de fournir des estimations plus précises de l'association entre environnement construit du quartier et activité physique et, par ricochet, de permettre d'élaborer des politiques et d'adopter des pratiques en matière d'urbanisation et de transport davantage aptes à fournir les améliorations souhaitées en matière d'activité physique.

Cette étude vise à estimer les associations relatives entre les caractéristiques de l'environnement construit des quartiers mesurées objectivement et la participation hebdomadaire ainsi que le temps consacré à différentes activités physiques réalisées dans le quartier, à savoir la marche comme mode de transport, la marche récréative, l'activité physique d'intensité modérée, l'activité physique d'intensité élevée et l'activité physique totale tout en tenant compte de l'autosélection résidentielle et des caractéristiques sociodémographiques.

\section{Méthodologie}

Des descriptions détaillées de la collecte des données et des analyses précédemment effectuées sont présentées ailleurs ${ }^{26,27}$. En bref, nous avons utilisé la composition aléatoire pour recruter deux échantillons transversaux indépendants d'adultes provenant de ménages situés dans la région urbaine de Calgary. Des entrevues téléphoniques ont été effectuées de juillet à octobre 2007 $(\mathrm{n}=2199$, taux de réponse $=33,6 \%)$ et répétées de janvier à avril $2008(n=2223$, taux de réponse $=36,7 \%$ ). Les deux échantillons, recrutés selon la même méthodologie, ont fourni des données sur les régimes d'activité physique l'été et l'hiver ${ }^{28}$. Les numéros de téléphone cellulaire n'ont pas été utilisés en complément de la liste de numéros de téléphone, car ils n'étaient pas faciles à obtenir pour les résidents de Calgary au moment de l'étude. Un adulte admissible et volontaire (18 ans et plus) de chaque ménage échantillonné a répondu aux questions de l'entrevue téléphonique portant, entre autres caractéristiques, sur l'activité physique, l'autosélection résidentielle, les variables sociodémographiques et le code postal du domicile. Le Comité conjoint d'éthique de la recherche en santé de l’Université de Calgary a approuvé l'étude.

\section{Variables}

\section{Environnement bâti du quartier}

Nous avons géocodé les codes postaux résidentiels à six chiffres en utilisant les coordonnées de longitude et de latitude du Fichier de conversion des codes postaux de Statistique Canada pour les utiliser dans la version 10 d'ArcGIS (Environmental Systems Research Institute, Inc., Redlands, Californie, États-Unis) afin de créer un polygone en réseau de type linéaire de 1,6 km de rayon (c'est-à-dire un " bassin marchant ») autour de la maison de chaque participant ${ }^{29,30}$. Les codes postaux ont été utilisés parce que les chercheurs ne disposaient pas des adresses de domicile complètes de tous les participants. Au Canada, les codes postaux urbains géocodés fournissent une estimation valide de la localisation géographique des ménages ${ }^{31}$. Dans les régions urbaines, les trois derniers chiffres d'un code postal 
indiquent un îlot urbain spécifique, c'est-àdire la zone d'un côté de la rue située entre deux rues qui se croisent ou un seul bâtiment, comme un grand immeuble à appartements. D’autres études ont également utilisé le bassin marchant de 1,6 km pour estimer les relations entre les caractéristiques de l'environnement bâti du quartier et l'activité physique ${ }^{32,33}$ : il s'agit de la distance approximative qu'un adulte type peut parcourir à pied (à une vitesse de $6,4 \mathrm{~km} / \mathrm{h}$ ) en 15 minutes environ.

Nous avons utilisé le logiciel ArcGIS avec les bases de données administratives municipales existantes pour estimer les caractéristiques de l'environnement bâti de chaque bassin marchant. Ces caractéristiques étaient (par kilomètre carré $\left[\mathrm{km}^{2}\right]$ ) les intersections, les entreprises et services autorisés, les arrêts d'autobus, la longueur de trottoir (en mètres) et l'offre de destinations récréatives. Nous avons utilisé les données correspondant aux limites du quartier administratif lorsque les données à l'échelle du bassin marchant n'étaient pas disponibles (population par $\mathrm{km}^{2}$, nombre d'arbres gérés par la ville par $\mathrm{km}^{2}$, longueur en mètres des sentiers ou pistes cyclables par $\mathrm{km}^{2}$ et superficie relative des espaces verts). Bien que notre objectif ait été d'étudier les neuf variables du milieu bâti à l'échelle du bassin marchant, comme ces données n'étaient pas disponibles à cette échelle pour toutes les variables de l'environnement bâti, nous avons décidé, plutôt que de les supprimer complètement de l'analyse, de conserver ces variables à l'échelle du quartier en raison de leurs associations potentielles avec l'activité physique. La sélection des variables liées au milieu bâti pour cette étude repose sur des données probantes antérieures ${ }^{12,13,26,27}$. Toutes les variables liées au milieu bâti ont été transformées en scores $\mathrm{z}$.

\section{Activité physique réalisée dans le quartier}

Les participants ont répondu à des questions adaptées du Neighbourhood Physical Activity Questionnaire (questionnaire sur l'activité physique dans le quartier) ${ }^{35}$ et validées lors d'un essai pilote ${ }^{34}$. On a demandé aux participants d'envisager quatre types d'activité physique, à savoir la marche comme moyen de transport, la marche récréative, l'activité physique d'intensité modérée et l'activité physique d'intensité élevée, effectuées dans un rayon de 15 minutes de marche de leur résidence. Pour la marche de transport, on a demandé aux participants d'indiquer combien de fois ils utilisaient la marche comme moyen de transport pour se rendre au travail et en revenir, aller faire des courses ou se rendre à un arrêt d'autobus ou de TLR (train léger sur rail) dans leur quartier ou aux alentours durant une semaine normale. Pour la marche récréative, on a demandé aux participants d'indiquer combien de fois ils marchaient pour le plaisir, la santé ou la forme (y compris promener le chien) dans leur quartier ou aux alentours durant une semaine normale. Les participants ont également indiqué le nombre total de minutes de marche de transport et de marche récréative effectuées dans leur quartier au cours d'une semaine normale. Les questions ont également porté sur l'activité physique d'intensité modérée effectuée dans le quartier (activité faite pour le plaisir, la santé ou la forme qui ne fait pas respirer fort ni s'essouffler) et l'activité physique d'intensité élevée (activité de mise en forme qui fait respirer plus fort ou qui essouffle).

Certaines données suggèrent qu'il peut y avoir des corrélats différents pour le démarrage d'une activité physique et pour son maintien $^{36}$. Nous avons donc estimé deux variables pour chaque type d'activité physique : (1) la non-participation (moins de 10 minutes/semaine) par rapport à la participation (10 minutes/semaine ou plus) et (2) la durée (minutes/semaine) chez ceux qui ont indiqué une participation. Les Nouvelles lignes directrices canadiennes en matière d'activité physique recommandent la pratique d'une activité physique modérée à intense par périodes d'au moins $10 \mathrm{minu}$ tes $^{37}$. Les minutes consacrées aux quatre types d'activité ont également été additionnées pour obtenir le nombre total de minutes d'activité physique hebdomadaire réalisée dans le quartier.

\section{Autosélection résidentielle}

Les participants ont indiqué l'importance ( " aucune », " faible » ou " grande ») d'une liste prédéterminée d'éléments expliquant le choix de leur quartier actuel comme lieu de résidence. En utilisant une analyse en composantes principales rapportée ailleurs $^{27}$, nous avons réparti ces 19 éléments dans 4 échelles d'autosélection du lieu de résidence : (1) accès à des endroits propices à l'activité physique (coefficient alpha de Cronbach $[\alpha]=0,79)$, (2) accès aux services locaux $(\alpha=0,61)$, (3) esprit de communauté $(\alpha=0,71)$ et (4) facilité à se déplacer en voiture $(\alpha=0,54)$. Les six éléments correspondant à l'échelle " Accès à des endroits propices à l'activité physique » révèlent l'importance de la proximité des parcs, de la proximité des installations récréatives, de la proximité des sentiers, l'existence de lieux où faire de l'activité physique, de lieux où se rendre à pied ou en vélo et de paysages attrayants (p. ex. montagnes). Quatre éléments correspondaient à l'échelle " Accès aux services locaux » : la facilité de marche, la proximité de l'école ou du travail, la proximité des transports en commun et la proximité des commerces ou des services. Quatre éléments (esprit de communauté, protection contre le crime, rues attrayantes et propreté des rues) formaient l'échelle " Esprit de communauté ». Deux éléments (l'importance de l'accès aux autoroutes et la facilité à se déplacer en voiture) ont été inclus dans l'échelle "Facilité à se déplacer en voiture ». Trois éléments décrivant l'importance de l'abordabilité, de la proximité du centre-ville et de la proximité des amis et de la famille n'ont été inclus dans aucune échelle et ont été ensuite retirés de l'analyse. Les réponses aux questions individuelles de chacune des quatre échelles ont été additionnées, les scores les plus élevés indiquant une préférence ou des raisons plus marquées pour choisir de résider dans le quartier en fonction de l'accès à des endroits propices à l'activité physique, de l'accès aux commerces et services locaux, de l'esprit de communauté et de la facilité à se déplacer en voiture.

\section{Caractéristiques sociodémographiques}

Les participants ont indiqué leur sexe, leur âge, le niveau de scolarité (école secondaire ou moins, collège, université), le nombre de personnes à charge de moins de 18 ans à la maison (aucune, un ou deux enfants et plus) et s'ils étaient propriétaires (ou en processus d'achat) ou locataires de leur résidence (propriétaire/acheteur ou non-propriétaire).

\section{Analyse statistique}

Nous avons effectué des statistiques descriptives, à savoir fréquences et mesures de la tendance centrale et de la variation (moyennes, écarts-types et médianes), pour les caractéristiques de l'environnement bâti, l'activité physique, l'autosélection de la résidence et les variables sociodémographiques. Nous avons estimé les corrélations de Pearson $(r)$ entre les neuf variables de l'environnement construit. Nous avons utilisé des modèles linéaires généralisés (distribution binomiale avec 
fonction de liaison logit) pour estimer les rapports de cotes (RC) et les intervalles de confiance (IC) à $95 \%$ de l'association entre la participation hebdomadaire habituelle et chaque caractéristique du milieu bâti, en tenant compte des covariables (autosélection de la résidence en fonction de l'accès aux occasions d'activité physique, de l'accès aux commerces et services, de l'esprit de communauté et de la facilité à se déplacer en voiture), des caractéristiques individuelles et de la saison de l'enquête pour évaluer la marche de transport, la marche récréative, l'activité physique d'intensité modérée et l'activité physique d'intensité élevée réalisées dans le quartier. Les modèles linéaires généralisés pondérés pour les covariables (distribution gamma avec fonction de lien identité) ont permis d'estimer l'association linéaire (coefficient $\beta$ [B] non normalisé et IC à $95 \%)$ entre les minutes hebdomadaires habituelles de marche de transport, de marche récréative, d'activité physique d'intensité modérée, d'activité physique d'intensité élevée et de l'ensemble de l'activité physique réalisées dans le quartier et chacune des neuf caractéristiques de l'environnement bâti. Nous avons évalué, pour les modèles entièrement ajustés, la qualité de l'ajustement en utilisant l'estimation du chi carré normatif ( $\mathrm{CN}$, modèle de chi carré/degrés de liberté). Les modèles ayant des valeurs $\mathrm{CN}$ inférieures ou égales à 2 ont été considérés comme ayant un ajustement acceptable. Les coefficients des modèles de valeurs $p$ inférieures à 0,05 ont été considérés comme statistiquement significatifs. Nous avons effectué nos analyses avec la version 22 de SPSS pour Windows (IBM Corp., Armonk, New York, É.-U.).

\section{Résultats}

Parmi les participants recrutés pour l'étude (n = 4 423), 4034 ont fourni des données complètes sur leur activité physique, l'autosélection résidentielle et leurs caractéristiques sociodémographiques. L'échantillon contenait un nombre plus important de femmes, d'universitaires, de personnes sans enfant à charge de moins de 18 ans et de propriétaires (tableau 1). L'âge moyen de l'échantillon était de 47,1 ans (écarttype de 15,6). En moyenne, les participants considéraient que l'accès à des possibilités d'activité physique, l'accès aux commerces et aux boutiques, l'esprit de communauté et la facilité de se déplacer en voiture étaient des raisons plutôt importantes expliquant leur choix de quartier actuel

TABLEAU 1

Échantillon sociodémographique, environnement bâti du quartier, autosélection du lieu de résidence et caractéristiques relatives à l'activité physique, Calgary, 2007-2008 $(n=4034)$

\begin{tabular}{|c|c|c|c|}
\hline & $\%$ & \multicolumn{2}{|c|}{ Moyenne (écart-type), médiane } \\
\hline \multicolumn{4}{|l|}{ Caractéristiques sociodémographiques } \\
\hline Âge (ans) & & $47,1(15,6)$ & 46,0 \\
\hline Sexe (femmes) & 59,7 & & \\
\hline \multicolumn{4}{|l|}{ Plus haut niveau de scolarité atteint } \\
\hline Diplôme d'études secondaires ou moins & 32,0 & & \\
\hline Collège & 26,0 & & \\
\hline Université & 42,0 & & \\
\hline \multicolumn{4}{|l|}{ Nombre d'enfants à la maison } \\
\hline Aucun & 62,7 & & \\
\hline Un ou plus & 37,2 & & \\
\hline Propriété résidentielle (propriétaires) & 81,5 & & \\
\hline \multicolumn{4}{|l|}{ Caractéristiques de l'environnement bâti du quartier } \\
\hline Intersections au km² & & 175,6 (26,6), & 174,4 \\
\hline Commerces au km²a & & $29,3(50,6)$, & 15,6 \\
\hline Arrêts d'autobus au km² a & & 13,8 (12,7), & 12,6 \\
\hline Variété des destinations récréatives au kmª & & $1,13(1,08)$, & 1,00 \\
\hline Longueur de trottoir $(\mathrm{m})$ au $\mathrm{km}^{2 \mathrm{a}}$ & & 16 140,4 (3 656,3), & 15690,0 \\
\hline Population totale au $\mathrm{km}^{2 \mathrm{~b}}$ & & 3 120,1 (1 652,6), & 2782,3 \\
\hline Proportion d'espaces verts $(\%)^{b}$ & & $18,0(10,0)$, & 15,0 \\
\hline Longueur de sentiers et de pistes cyclables $(\mathrm{m})$ au $\mathrm{km}^{2 \mathrm{~b}}$ & & $2464,4(1438,6)$, & 2391,7 \\
\hline Arbres entretenus par la ville au $\mathrm{km}^{2 \mathrm{~b}}$ & & $1586,1(515,1)$ & 1658,3 \\
\hline \multicolumn{4}{|l|}{ Raisons de l'autosélection du lieu de résidence } \\
\hline Accès aux possibilités d'activité physique & & $2,0(0,5)$ & 2,0 \\
\hline Accès aux commerces et aux services & & $2,1(0,5)$ & 2,2 \\
\hline Esprit communautaire & & $2,4(0,5)$ & 2,5 \\
\hline Facilité à se déplacer en véhicule motorisé & & $2,1(0,6)$ & 2,0 \\
\hline \multicolumn{4}{|c|}{ Pratique d'une activité physique et le nombre de minutes au cours d'une semaine type ${ }^{\text {d }}$} \\
\hline Marche comme moyen de transport dans le quartier & 59,1 & $121,2(146,0)$ & $60,0^{\mathrm{e}}$ \\
\hline Marche récréative dans le quartier & 74,9 & 186,2 (177,6), & $120,0^{\mathrm{e}}$ \\
\hline AP d'intensité modérée dans le quartier & 35,5 & $141,1(148,9)$ & $90,0^{\mathrm{e}}$ \\
\hline AP d'intensité élevée dans le quartier & 45,5 & $171,5(157,5)$ & $120,0^{\mathrm{e}}$ \\
\hline AP totale dans le quartier & $91,2^{f}$ & $372,2(344,3)$, & $270,0^{e}$ \\
\hline
\end{tabular}

Abréviations : AP, activité physique; $\mathrm{km}^{2}$, kilomètres carrés; $\mathrm{m}$, mètres.

a Estimé pour le bassin marchant de 1,6 km.

${ }^{\mathrm{b}}$ Estimé pour la frontière administrative du quartier.

' Les scores les plus élevés représentent l'augmentation de l'importance du facteur relatif au choix du quartier.

¿ Les participants pouvaient déclarer plus d'un type d'activité physique.

${ }^{e}$ Estimation fondée sur ceux qui ont déclaré pratiquer une activité physique (c.-à-d. 10 minutes ou plus par semaine).

${ }_{\mathrm{f}}^{\mathrm{f}}$ Pourcentage de l'échantillon qui indique pratiquer au moins l'une des activités physiques indiquées ci-dessus.

(tableau 1). Les corrélations de Pearson entre les neuf variables de l'environnement bâti variaient entre $r=-0,30$ (entre la proportion d'espaces verts et la population $/ \mathrm{km}^{2}$ ) à $r=0,62$ (entre le nombre d'entreprises $/ \mathrm{km}^{2}$ et d'arrêts d'autobus/ $\mathrm{km}^{2}$ ). Sur les 36 corrélations estimées parmi les variables du milieu bâti, toutes sauf 5 étaient inférieures à $\pm 0,30$ (résultats non présentés). 


\section{Participation hebdomadaire à une activité physique dans le quartier}

Plus de la moitié des participants ont déclaré avoir pratiqué la marche dans leur quartier comme mode de transport $(59,1 \%)$ ou comme loisir (74,9\%) (tableau 1). Ils ont été moins nombreux à déclarer s'adonner à une activité physique d'intensité modérée $(35,5 \%)$ et à une activité physique d'intensité élevée (45,5\%). Après pondération des covariables, on a constaté que la marche comme mode de transport était positivement associée à la densité des intersections $(\mathrm{RC}=1,11$; IC à $95 \%: 0,5 \%$ 1,03 à 1,20), à la densité commerciale $(1,52 ; 1,29$ à 1,78$)$ et à la longueur de trottoir $(1,19 ; 1,09$ à 1,29) (tableau 2). La longueur de trottoir était également associée positivement à la participation à une activité physique dans le quartier d'intensité modérée $(1,11 ; 1,02$ à 1,19$)$ et d'intensité élevée $(1,11 ; 1,02$ à 1,20$)$. Après pondération des covariables, la longueur des sentiers ou pistes cyclables était associée positivement à la marche récréative dans le quartier $(1,17 ; 1,05$ à 1,31$)$ et à l'activité physique d'intensité élevée $(1,12 ; 1,02$ à
1,24). En outre, l'offre de destinations récréatives était associée positivement à la participation à une activité physique d'intensité modérée dans le quartier (1,09; 1,01 à 1,17). La proportion d'espaces verts dans le quartier était négativement associée à la participation à une activité physique d'intensité élevée $(0,89 ; 0,82$ à 0,98$)$ (tableau 2). À titre de comparaison, les associations estimées entre la participation à une activité physique dans le quartier et les caractéristiques de l'environnement bâti sans ajustement de l'autosélection du lieu de résidence sont présentées dans le tableau 3.

\section{Minutes hebdomadaires d'activité physique dans le quartier}

Pour les personnes ayant déclaré une participation, le nombre moyen de minutes était plus élevé pour la marche récréative de quartier (186,2 \pm 177,6 minutes/ semaine), puis pour l'activité physique intense (171,5 $\pm 157,5$ minutes/semaine), l'activité physique d'intensité modérée $(141,1 \pm 148,9$ minutes/semaine) et la marche récréative $(121,2 \pm 146,0$ minutes/ semaine) (tableau 1). Avec la pondération des covariables, la marche pour le transport dans le quartier était significativement $(p<0,05)$ associée à la densité commerciale ( $\mathrm{B}=19,24$ minutes/semaine, IC à $95 \%$ : 11,28 à 27,20), à l'offre de destinations récréatives $(-8,88$ minutes/semaine, $-12,49$ à $-5,28)$ et à la densité des arbres gérés par la ville $(6,15$ minutes/semaine; 2,29 à 10,72) (tableau 4). De plus, la densité de population était associée négativement à l'activité physique d'intensité modérée dans le quartier ( $-8,65$ minutes/ semaine, $-15,32$ à -1,98). La densité des arbres gérés par la ville était associée positivement à l'activité physique d'intensité élevée dans le quartier (7,28 minutes/ semaine, 0,39 à 14,17). En particulier, seules la densité commerciale (27,35 minutes/ semaine; 9,86 à 44,83 ) et la longueur de trottoir $(18,69$ minutes/semaine; 7,69 à 29,69 ) étaient associées à l'activité physique totale dans le quartier. Aucune caractéristique du milieu bâti n'était associée de façon significative au nombre de minutes hebdomadaires de marche récréative (tableau 4). À titre de comparaison, les associations estimées entre le nombre de minutes

TABLEAU 2

Modèle linéaire généralisé (loi binominale et fonction de liaison logit) pour estimation des RC et des IC à $95 \%$ de l'association entre la pratique d'une activité physique axée sur le quartier et les caractéristiques de l'environnement bâti, Calgary, 2007-2008 (n = 4 034)

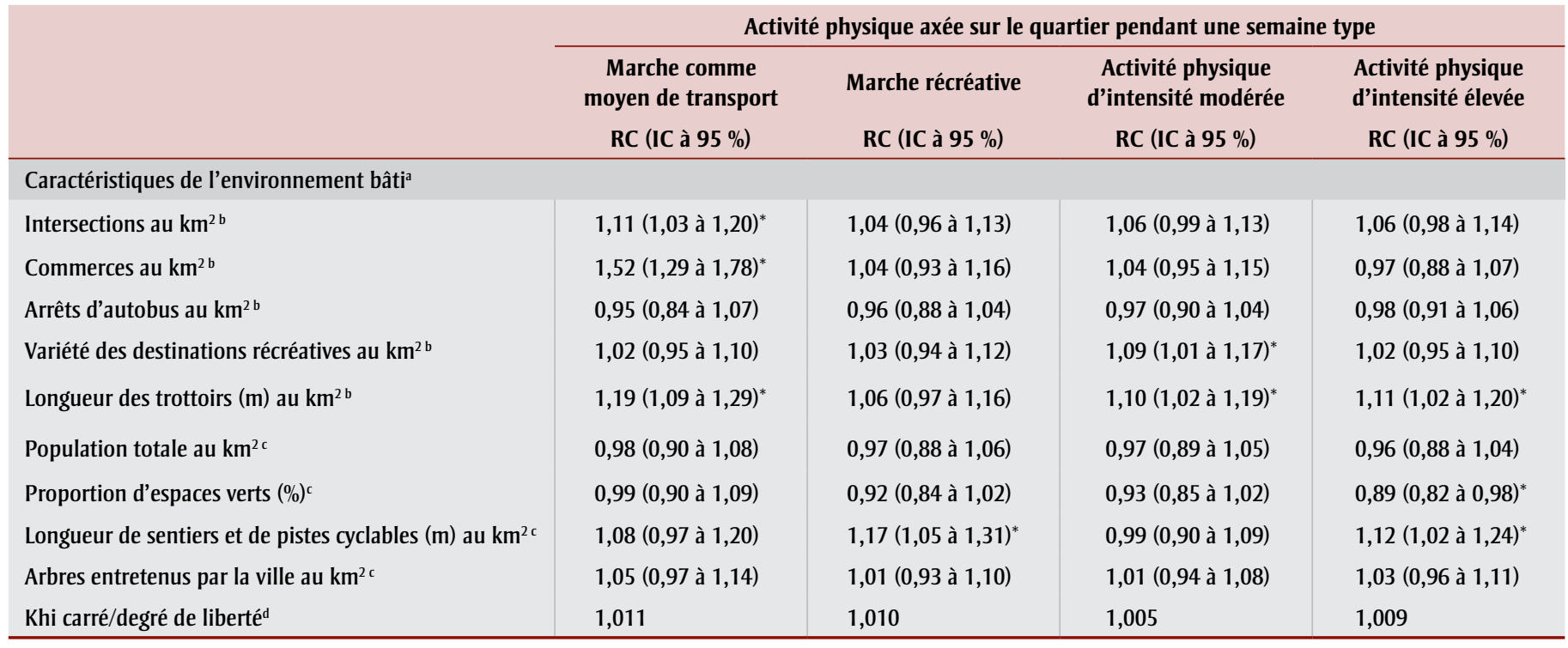

Abréviations : IC, intervalle de confiance; km², kilomètres carrés; m, mètres; RC, rapport de cotes.

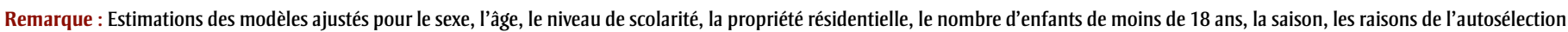
du lieu de résidence et les caractéristiques de l'environnement bâti.

a Toutes les variables relatives à l'environnement bâti sont normalisées (score z).

${ }^{\mathrm{b}}$ Estimation relative au bassin marchant de $1,6 \mathrm{~km}$.

c Estimation liée aux frontières administratives du quartier.

${ }^{d}$ Les valeurs les plus près de 1 représentent une meilleure qualité de l'ajustement. La qualité de l'ajustement est basée sur le modèle entièrement ajusté. ${ }^{*} p<0,05$. 
TABLEAU 3

Modèle linéaire généralisé (loi binominale et fonction de liaison logit) pour estimation des RC et IC à $95 \%$ de l'association entre la pratique d'une activité physique axée sur le quartier et les caractéristiques de l'environnement bâti, sans ajustement des variables relatives à l'autosélection du lieu de résidence, Calgary, 2007-2008 ( $\mathrm{n}=4$ 034)

\begin{tabular}{|c|c|c|c|c|}
\hline & \multicolumn{4}{|c|}{ Activité physique axée sur le quartier pendant une semaine type } \\
\hline & $\begin{array}{c}\text { Marche comme } \\
\text { moyen de transport } \\
\text { RC (IC à } 95 \%)\end{array}$ & $\begin{array}{l}\text { Marche récréative } \\
\text { RC (IC à } 95 \%)\end{array}$ & $\begin{array}{l}\text { Activité physique } \\
\text { d'intensité modérée } \\
\text { RC (IC à } 95 \% \text { ) }\end{array}$ & $\begin{array}{l}\text { Activité physique } \\
\text { d'intensité élevée } \\
\text { RC (IC à } 95 \% \text { ) }\end{array}$ \\
\hline \multicolumn{5}{|l|}{ Caractéristiques de l'environnement bâti ${ }^{a}$} \\
\hline Intersections au $\mathrm{km}^{2 \mathrm{~b}}$ & $1,10(1,02 \text { à } 1,18)^{*}$ & $0,98(0,90$ à 1,06$)$ & $1,03(0,96$ à 1,10$)$ & $1,02(0,95$ à 1,09$)$ \\
\hline Commerces au km² & $1,67(1,42 \text { à } 1,95)^{*}$ & $1,04(0,93$ à 1,16$)$ & $1,06(0,96$ à 1,16$)$ & $1,00(0,91$ à 1,10$)$ \\
\hline Variété des destinations récréatives au $\mathrm{km}^{2 \mathrm{~b}}$ & $1,06(0,98$ à 1,14$)$ & $1,03(0,95$ à 1,12$)$ & $1,10(1,02 \text { à } 1,18)^{*}$ & $1,04(0,97$ à 1,12$)$ \\
\hline Longueur des trottoirs $(\mathrm{m}) \mathrm{au} \mathrm{km}^{2 \mathrm{~b}}$ & $1,21(1,12$ à 1,32$)$ & $1,02(0,94$ à 1,11$)$ & $1,08(1,00 \text { à } 1,16)^{*}$ & $1,08(1,00 \text { à } 1,16)^{*}$ \\
\hline Population totale au $\mathrm{km}^{2 \mathrm{c}}$ & $0,98(0,90$ à 1,07$)$ & $0,92(0,84$ à 1,01$)$ & $0,94(0,87$ à 1,02$)$ & $0,93(0,86$ à 1,00$)$ \\
\hline Proportion d'espaces verts (\%) c & $0,99(0,90$ à 1,08$)$ & $0,92(0,83$ à 1,01$)$ & $0,93(0,85$ à 1,02$)$ & $0,90(0,82 \text { à } 0,98)^{*}$ \\
\hline
\end{tabular}

Abréviations : IC, intervalle de confiance; $\mathrm{km}^{2}$, kilomètres carrés; m, mètres; RC, rapport de cotes.

Remarque : Estimations des modèles ajustés pour le sexe, l'âge, le niveau de scolarité, la propriété résidentielle, le nombre d'enfants de moins de 18 ans et la saison.

a Toutes les variables relatives à l'environnement bâti sont normalisées (score z).

${ }^{\mathrm{b}}$ Estimation relative au bassin marchant de $1,6 \mathrm{~km}$.

' Estimation relative aux frontières administratives du quartier.

${ }^{\text {d} L e s ~ v a l e u r s ~ l e s ~ p l u s ~ p r e ̀ s ~ d e ~} 1$ représentent une meilleure qualité de l'ajustement. La qualité de l'ajustement est basée sur le modèle entièrement ajusté.

${ }^{*} p<0,05$.

hebdomadaires d'activité physique dans le quartier et les caractéristiques du milieu bâti sans correction pour l'autosélection résidentielle sont présentées au tableau 5.

\section{Analyse}

Les résultats de notre étude montrent que différentes caractéristiques de l'environnement bâti du quartier mesurées objectivement sont associées à différents types d'activité physique chez les adultes. Comme l'ont déjà souligné d'autres données probantes ${ }^{12,14,15}$, les caractéristiques de l'environnement bâti du quartier semblent plus importantes dans le contexte de la marche comme moyen de transport que dans celui de la marche récréative. À l'instar d'autres études, nous avons trouvé que les caractéristiques de l'environnement bâti du quartier associées à l'activité physique d'intensité modérée ne sont pas les mêmes que celles associées à l'activité physique d'intensité élevée ${ }^{8,13,16}$. Par ailleurs, ces résultats donnent à penser que la corrélation entre l'environnement bâti et la participation à l'activité physique (c.-à-d. 10 minutes ou plus par semaine contre moins de 10 minutes par semaine) pourrait ne pas être la même que celle entre l'environnement bâti et le temps passé à faire de l'activité physique. Ces résultats sont nouveaux dans le sens où nous avons évalué les liens entre l'environnement bâti et différents types d'activité physique axée sur le quartier tout en procédant à un ajustement statistique de l'autosélection du lieu de résidence, et dans le sens où nous avons examiné à la fois la participation à l'activité physique et la durée de celle-ci en tant que résultats distincts.

Nous avons observé qu'un plus grand nombre de caractéristiques de l'environnement bâti étaient significativement associées à la marche comme moyen de transport qu'à la marche récréative. Nous avons trouvé que la connectivité (densité des intersections), le nombre de destinations (densité des commerces) et l'accès à des trottoirs (la longueur de trottoir) étaient associés de façon positive à la marche comme moyen de transport, comme dans d'autres études ${ }^{12,38}$. Soulignons que nos résultats indiquent que l'accroissement de la densité de commerces dans les quartiers pourrait entraîner à la fois l'augmentation du nombre de personnes qui commencent à utiliser la marche comme moyen de transport et le temps global passé à pratiquer ce type de marche. Nous avons également constaté que la densité des arbres entretenus par la ville était associée au temps passé à pratiquer la marche comme moyen de transport à l'intérieur du quartier. Il s'agit là d'un résultat inattendu étant donné que le paysage (notamment les jardins et les arbres) est habituellement associé à l'activité physique récréative ${ }^{39}$. Nous supposons que la densité d'arbres est probablement plus élevée dans les quartiers plus vieux, lesquels offrent habituellement une infrastructure plus propice à la marche comme moyen de transport ${ }^{40,41}$. Une seule caractéristique de l'environnement bâti - la longueur des sentiers et des voies cyclables par $\mathrm{km}^{2}$ - était associée à la pratique de la marche récréative dans le quartier, bien que cette caractéristique ne semble pas liée au temps passé à pratiquer la marche récréative axée sur le quartier.

La variété de destinations récréatives par $\mathrm{km}^{2}$ était associée de façon positive à la pratique d'une activité physique d'intensité modérée, mais pas au nombre de minutes. 
TABLEAU 4

Modèle linéaire généralisé (distribution Gamma et fonction de lien identité) pour estimation non normalisée du coefficient bêta (B) et IC à $95 \%$ de l'association entre le temps passé à pratiquer une activité physique axée sur le quartier et les caractéristiques de l'environnement bâti, Calgary, 2007-2008

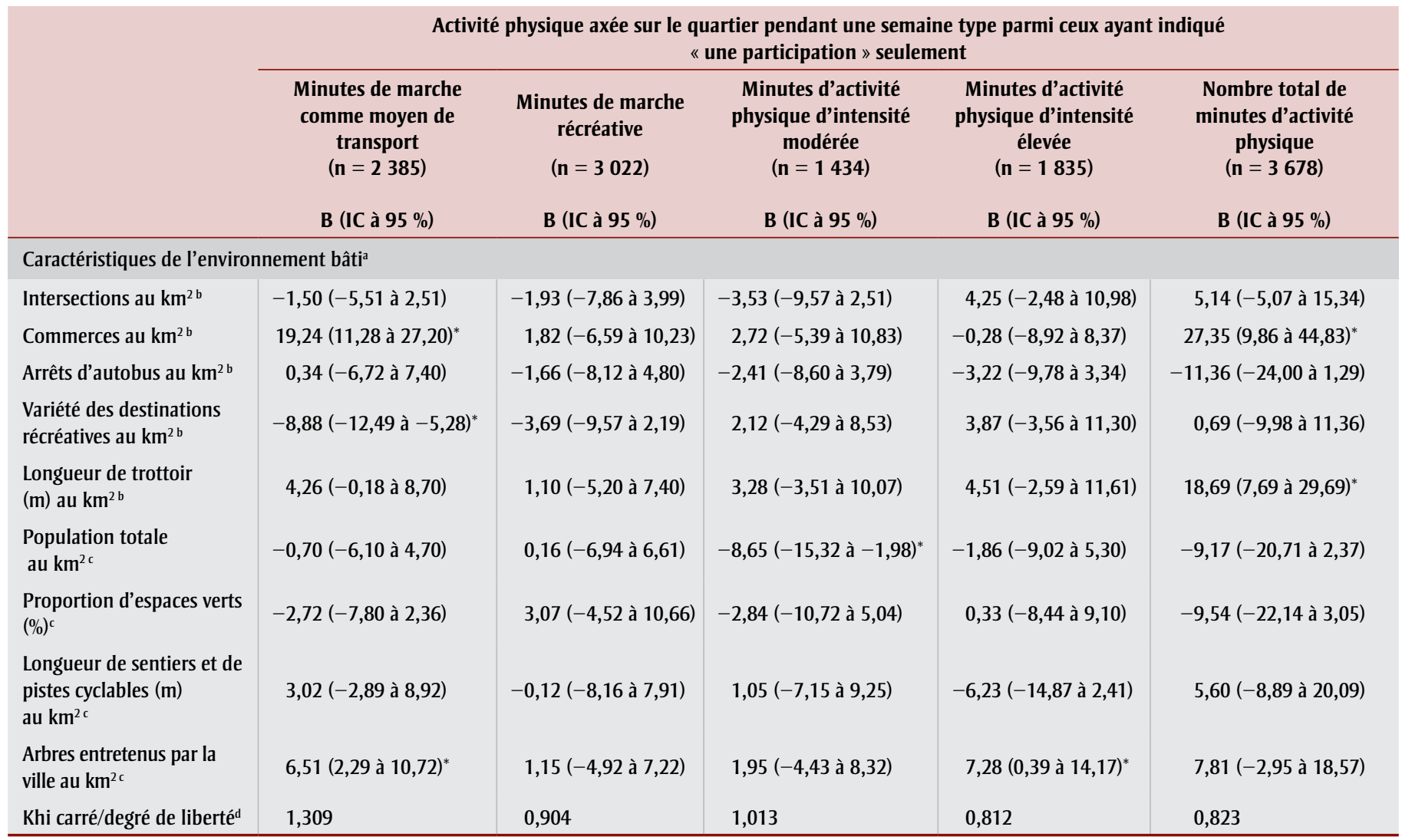

Abréviations : B, coefficient bêta non normalisé; IC, intervalle de confiance; km², kilomètres carrés; $\mathrm{m}$, mètres.

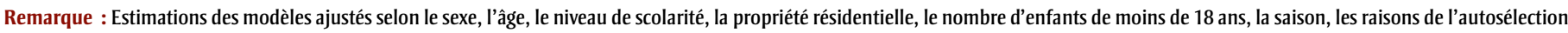
du lieu de résidence (accès aux possibilités d'activité physique, accès aux services et aux commerces, esprit communautaire, facilité à se déplacer en véhicule motorisé).

${ }^{a}$ Toutes les variables relatives à l'environnement bâti sont normalisées (score z).

${ }^{\mathrm{b}}$ Estimation relative au bassin marchant de 1,6 km.

${ }^{c}$ Estimation relative aux frontières administratives du quartier.

${ }^{d}$ Les valeurs les plus près de 1 représentent une meilleure qualité de l'ajustement. La qualité de l'ajustement est basée sur le modèle entièrement ajusté.

${ }^{*} p<0,05$.

La variété de destinations récréatives était par contre associée de façon négative au nombre de minutes passées à pratiquer la marche comme moyen de transport dans le quartier. Une des explications à cette situation pourrait être que les destinations récréatives et non récréatives sont en concurrence sur le plan de l'espace géographique, ce qui fait que plus le nombre d'installations récréatives est élevé, moins il y a d'espace pour les destinations utilitaires où il est possible de se rendre à pied. Malgré les données prouvant l'importance des parcs pour favoriser l'activité physique $^{42,43,44}$, nous n'avons trouvé aucune association positive significative entre la proportion d'espaces verts et l'un ou l'autre de nos résultats quant à l'activité physique. Étonnamment, nous avons trouvé qu'une proportion élevée d'espaces verts dans un quartier était associée à une plus faible possibilité de participation à une activité physique d'intensité élevée et axée sur le quartier. D'autres ont prouvé que la présence de parcs et d'espaces en plein air et la qualité de ces derniers étaient associées de façon positive à la course à pied ${ }^{18}$. Nous n'avons pas été en mesure d'établir une distinction entre les différents types d'activité physique d'intensité élevée possibles. Remarquons que notre mesure liée aux espaces verts ne faisait pas la distinction entre les parcs en fonction de leur qualité, de leur type ni de leur taille, trois facteurs susceptibles de compter parmi les caractéristiques importantes qu'on associe à l'activité physique dans les parcs ${ }^{45,46}$, et qu'elle comprenait les espaces verts délimitant les frontières administratives des quartiers, et n'était donc pas spécifique au bassin marchant de 1,6 km. Pour bien des adultes, les parcs ne constituent pas une destination importante en soi pour la pratique d'une activité physique d'intensité élevée, mais plutôt une destination incluse dans le trajet parcouru à la course ou à vélo ${ }^{47}$. Bien que nous ayons tenté d'ajuster les données quant à l'autosélection du lieu de résidence, il est possible que certaines personnes enclines à pratiquer une activité physique d'intensité élevée choisissent aussi d'habiter dans un quartier où il $\mathrm{y} a$ moins d'espaces verts.

Des études antérieures ont trouvé que l'accès à des trottoirs est important pour 


\section{TABLEAU 5}

Modèle linéaire généralisé (distribution Gamma et fonction de lien identité) pour estimation non normalisée du coefficient bêta (B) et des IC à 95 \% de l'association entre le temps passé à pratiquer une activité physique axée sur le quartier et les caractéristiques de l'environnement bâti, sans ajustement des variables relatives à l'autosélection du lieu de résidence, Calgary, 2007-2008

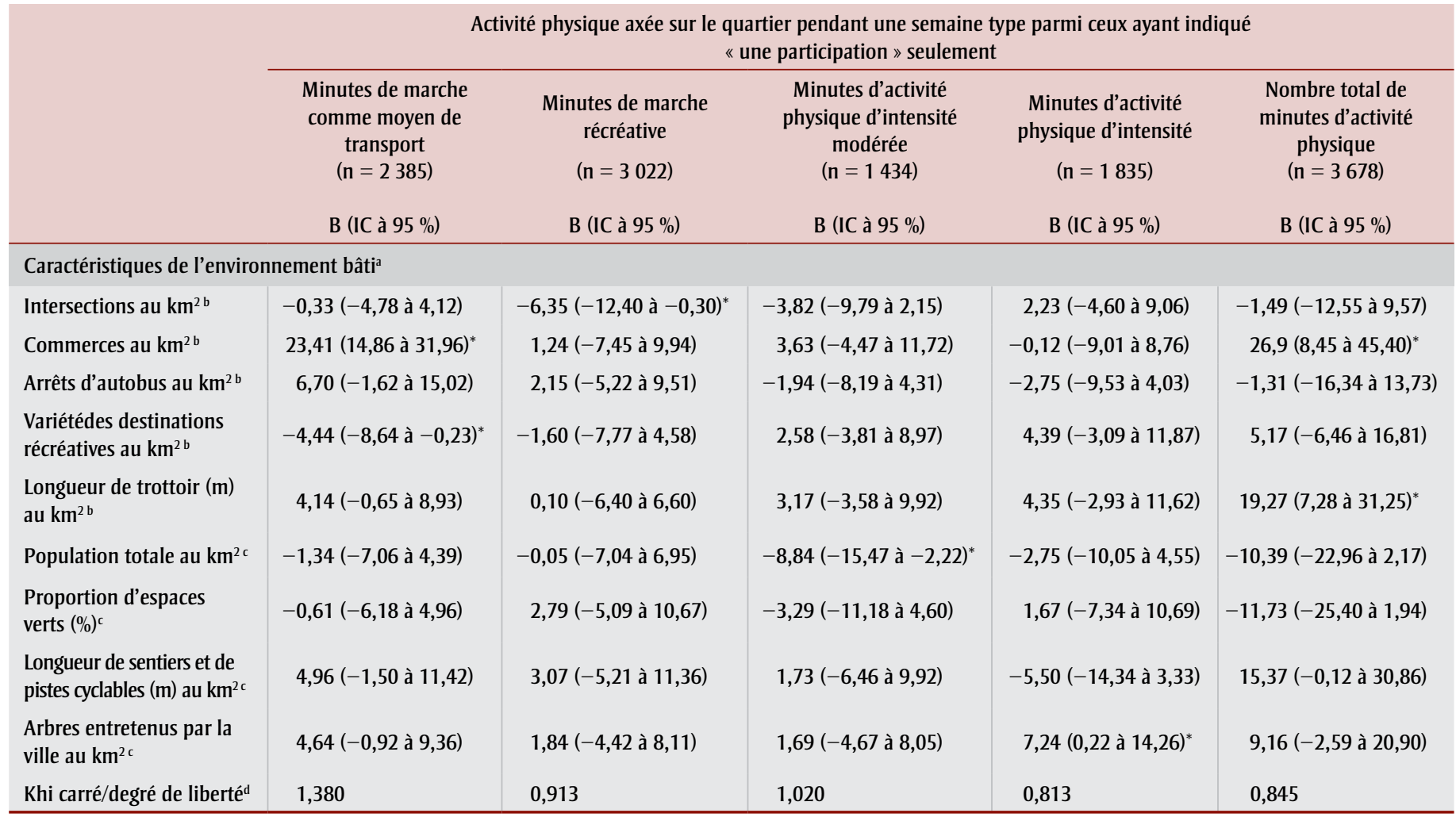

Abréviations : $\mathrm{B}$, coefficient bêta non normalisé; IC, intervalle de confiance; km², kilomètres carrés; m, mètres.

Remarque : Estimations des modèles ajustés selon le sexe, l’âge, le niveau de scolarité, la propriété résidentielle, le nombre d'enfants de moins de 18 ans et la saison.

a Toutes les variables relatives à l'environnement bâti sont normalisées (score z).

${ }^{\mathrm{b}}$ Estimation relative au bassin marchant de $1,6 \mathrm{~km}$.

' Estimation relative aux frontières administratives du quartier.

${ }^{\mathrm{d}}$ Les valeurs les plus près de 1 représentent une meilleure qualité de l'ajustement. La qualité de l'ajustement est basée sur le modèle entièrement ajusté.

${ }^{*} p<0,05$.

favoriser la marche comme moyen de transport $^{17,48}$ ainsi que l'activité d'intensité modérée à élevée ${ }^{17}$. Notre étude indique aussi que les trottoirs sont associés de façon positive à la marche comme moyen de transport et à l'activité physique en général à l'intérieur du quartier. Davantage de trottoirs dans les quartiers déjà construits pourrait s'avérer un moyen économique de promouvoir la marche comme moyen de transport ${ }^{49}$. Nos résultats indiquent que la présence de trottoirs pourrait également favoriser la pratique d'autres types d'activité physique axées sur le quartier de différentes intensités, ce qui pourrait s'avérer avantageux du point de vue de la santé. Précisons que le nombre de destinations commerciales et la longueur des trottoirs sont les deux seules caractéristiques significativement associées au nombre total de minutes à pratiquer une activité physique axée sur le quartier. L'augmentation d'un écart-type de la densité commerciale était associée à une augmentation de 25 minutes d'activité physique axée sur le quartier par semaine alors que l'augmentation d'un écart-type de la longueur des trottoirs était associée à une augmentation de 18 minutes d'activité physique axée sur le quartier par semaine. Du point de vue de la planification et comparativement à d'autres caractéristiques de l'environnement bâti, les trottoirs pourraient s'avérer moins difficiles et moins coûteux à modifier dans les limites des contraintes de l'infrastructure des quartiers existants. Modifier les ordonnances de zonage pour favoriser l'établissement d'autres commerces et services combinés à l'aménagement du territoire dans les nouveaux et les anciens quartiers pourrait contribuer à la hausse de la pratique d'activités physiques axées sur le quartier. Il convient de noter que malgré certaines associations négatives entre les caractéristiques de l'environnement bâti et la pratique de certaines activités physiques (c.-à-d. la densité de la population et l'activité physique d'intensité modérée, la densité des destinations récréatives et la marche comme moyen de transport ainsi que la proportion d'espaces verts et la pratique d'activité physique d'intensité élevée), aucune caractéristique de l'environnement bâti ne s'est avérée associée de façon négative et importante à la pratique d'activité physique dans son ensemble. Par conséquent, les travaux 
visant à rendre l'environnement bâti d'un quartier plus favorable à la pratique de l'activité physique sont susceptibles de ne donner lieu à aucun changement ou de donner lieu à une augmentation, et non à une diminution, de la pratique d'activités physiques axées sur le quartier en général.

\section{Forces et limites}

La présente étude a plusieurs forces, en particulier l'association entre environnement bâti et activité physique axée sur le quartier telle que nous l'avons définie (c.à-d. toute destination située à 15 minutes ou moins de marche du domicile), l'ajustement statistique de l'autosélection du lieu de résidence et enfin l'évaluation des associations relatives entre différentes caractéristiques de l'environnement bâti et quatre comportements associés à la pratique de l'activité physique ainsi qu'avec l'activité physique axée sur le quartier en général.

En dépit de ces forces, l'utilisation de données autodéclarées sur la pratique de l'activité physique constitue l'une des limites de notre étude en raison de la possibilité d'erreurs dans les mesures ${ }^{50}$. Pour notre étude, nous avons envisagé l'utilisation d'une mesure objective de l'activité physique, comme l'accéléromètre, mais elle est plus difficile à mettre en application que l'autodéclaration. Il convient de noter que nos estimations quant à la durée de la pratique d'une activité physique chaque semaine étaient supérieures à ce à quoi on pourrait s'attendre au sein de cette population, ce qui est peut-être attribuable au fait que les non-participants ne font pas partie de ces estimations.

Le taux de réponse peut restreindre la généralisation de nos résultats. Comparativement à la population de Calgary, les répondants au sondage téléphonique étaient plus instruits, comptaient une plus grande proportion d'aînés (60 ans et plus), étaient plus susceptibles d'avoir des personnes à charge de moins de 18 ans et comptaient une proportion plus importante de femmes, de personnes nées au Canada et de propriétaires $^{28}$. Ajoutons que seuls les ménages ayant un téléphone fixe pouvaient être recrutés dans le cadre de notre étude. Sur le plan de la santé et des caractéristiques sociodémographiques, il peut y avoir des différences entre les ménages disposant d'un téléphone fixe et ceux n'en disposant pas $^{51}$.
Malgré l'ajustement statistique des données relatives à l'autosélection du lieu de résidence, nos données transversales ne permettent pas de déterminer le rapport de causalité entre l'environnement bâti et l'activité physique. La plupart des estimations quant à l'association entre variables de l'environnement bâti et activité physique demeuraient inchangées ou s'atténuaient légèrement à la suite de l'ajustement des variables relatives à l'autosélection du lieu de résidence. Une seule association significative sur le plan statistique avant l'ajustement des données relatives à l'autosélection du lieu de résidence s'est atténuée et ne s'est pas avérée significative sur le plan statistique après cet ajustement : la densité des intersections en lien avec le nombre de minutes de marche récréative. Ces résultats donnent à penser que si l'ajustement des données relatives à l'autosélection du lieu de résidence dans les études transversales portant sur l'environnement bâti et l'activité physique est important, les répercussions sur l'estimation des associations peuvent s'avérer minimes et, dans la plupart des cas, n'avoir aucune incidence sur les conclusions. Il faudra mener des expériences naturelles pour évaluer les relations temporelles entre les changements dans l'environnement bâti et les changements quant à la pratique de l'activité physique ${ }^{52}$.

Le problème du changement d'unité spatiale suppose que la définition opérationnelle choisie pour décrire le quartier peut avoir une incidence sur les associations estimées en lien avec l'activité physique $^{53}$. Les définitions quant aux limites du quartier peuvent avoir une influence sur les associations estimées entre l'environnement bâti et l'activité physique $^{54,55}$. Il est possible que les associations faite avec un bassin marchant de 1,6 km ne puissent pas être généralisées à d'autres délimitations de l'étendue du bassin marchant. L'utilisation d'un système de localisation GPS associée à l'utilisation d'accéléromètres constitue une approche prometteuse grâce à laquelle rendre compte des comportements qui définissent les quartiers et la pratique d'activité physique chez chaque individu ${ }^{56}$. Nos mesures de l'environnement bâti, bien que globales, ne sont pas exhaustives et ne tiennent pas compte de l'échelle micro ni des caractéristiques du paysage urbain. Par exemple, l'accès aux transports en commun n'était représenté que par la densité des arrêts d'autobus dans le quartier alors que le fait que les services de transport en train, qu'il s'agisse d'escales ou non, offrent un abri contre les intempéries, l'horaire des circuits et la fréquence du service, entre autres facteurs, peut aussi peser dans la balance au moment de décider d'utiliser la marche comme moyen de transport.

\section{Conclusion}

Soulignons que nos résultats font état de liens entre environnement bâti du quartier et activité physique axée sur le quartier, même après ajustement pour des raisons liées à l'autosélection du lieu de résidence. Par ailleurs, nous avons obtenu des données probantes quant aux corrélats des comportements spécifiques à l'environnement bâti du quartier ${ }^{24}$. Des modifications apportées à certaines caractéristiques de l'environnement bâti peuvent ne pas avoir le même effet sur toutes les activités physiques. À ce sujet, certaines des caractéristiques de l'environnement bâti peuvent être plus efficaces que d'autres pour promouvoir ou faciliter l'entame de la pratique d'une activité physique ou la participation à cette dernière alors que d'autres caractéristiques de l'environnement bâti semblent favoriser le maintien ou l'augmentation du temps passé à pratiquer une activité physique chez les personnes déjà actives. Nos résultats laissent penser que, pour accroître la pratique globale d'activité physique axée sur le quartier, les urbanistes pourraient prendre plus particulièrement en considération l'augmentation de la densité des destinations commerciales (utilitaires) et le nombre de trottoirs ou la longueur en trottoirs. L'incidence des caractéristiques de l'environnement bâti sur différents types d'activité physique devrait faire partie des considérations au moment de planifier, de concevoir et de modifier l'environnement bâti du quartier.

\section{Remerciements}

La présente étude fait partie du projet EcoEUFORIA financé par les Instituts de recherche en santé du Canada (IRSC; Alan Shiell, Ph. D., chercheur principal). Gavin McCormack bénéficie du soutien de la bourse salariale de nouveau chercheur de l'IRSC.

\section{Conflits d'intérêts}

L'auteur déclare n'avoir aucun conflit d'intérêts. 


\section{Références}

1. Statistique Canada. Activité physique directement mesurée chez les adultes, 2012 et 2013 [Internet]. Ottawa (Ont.) : Statistique Canada; [modifié le 27 nov. 2015; consulté en juin 2016]. En ligne à : http://www.statcan.gc.ca/pub/82 -625-x/2015001/article/14135-fra.htm

2. Colley RC, Garriguet D, Janssen I, Craig CL, Clarke J, Tremblay MS. Activité physique des adultes au Canada : résultats d'accélérométrie de l'Enquête canadienne sur les mesures de la santé de 2007-2009. Rapports sur la santé. 2011; 22(1):7-15.

3. Shiroma EJ, Sesso HD, Moorthy MV, Buring JE, Lee IM. Do moderate-intensity and vigorous-intensity physical activities reduce mortality rates to the same extent? J Am Heart Assoc [Internet]. 2014;3(5):e000802. En ligne à : http://jaha.ahajournals.org/content /3/5/e000802

4. Chomistek AK, Cook NR, Flint AJ, Rimm EB. Vigorous-intensity leisuretime physical activity and risk of major chronic disease in men. Med Sci Sports Exerc. 2012;44(10):1898-1905.

5. Samitz G, Egger M, Zwahlen $M$. Domains of physical activity and allcause mortality: systematic review and dose-response meta-analysis of cohort studies. Int J Epidemiol. 2011; 40(5):1382-1400.

6. Lollgen H, Bockenhoff A, Knapp G. Physical activity and all-cause mortality: an updated meta-analysis with different intensity categories. Int J Sports Med. 2009;30(3):213-224.

7. Swain DP, Franklin BA. Comparison of cardioprotective benefits of vigorous versus moderate intensity aerobic exercise. Am J Cardiol. 2006;97(1): 141-147.

8. Salvo D, Reis RS, Hino AA, Hallal PC, Pratt M. Intensity-specific leisure-time physical activity and the built environment among Brazilian adults: a bestfit model. J Phys Act Health. 2015; 12(3):307-318.

9. Warburton DE, Bredin SS. Reflections on physical activity and health: what should we recommend? Can J Cardiol. 2016;32(4):495-504.
10. Janssen I. Health care costs of physical inactivity in Canadian adults. Appl Physiol Nutr Metab. 2012;37(4):803806.

11. Trost SG, Owen N, Bauman AE, Sallis JF, Brown W. Correlates of adults' participation in physical activity: review and update. Med Sci Sports Exerc. 2002;34(12):1996-2001.

12. Saelens B, Handy S. Built environment correlates of walking: a review. Med Sci Sports Exerc. 2008;40(7 Suppl): S550-S566.

13. Wendel-Vos W, Droomers M, Kremers $\mathrm{S}$, Brug J, van Lenthe F. Potential environmental determinants of physical activity in adults: a systematic review. Obes Rev. 2007;8(5):425-440.

14. McCormack G, Shiell A. In search of causality: a systematic review of the relationship between the built environment and physical activity among adults. Int $\mathrm{J}$ Behav Nutr Phys Act [Internet]. 2011 Nov 13;8(1):125. En ligne à : http://dx.doi.org/10.1186 /1479-5868-8-125

15. Coogan PF, White LF, Adler TJ, Hathaway KM, Palmer JR, Rosenberg L. Prospective study of urban form and physical activity in the Black Women's Health Study. Am J Epidemiol. 2009;170(9):1105-1117.

16. De Bourdeaudhuij I, Sallis JF, Saelens BE. Environmental correlates of physical activity in a sample of Belgian adults. Am J Health Promot. 2003; 18(1):83-92.

17. Giles-Corti B, Donovan R. Socioeconomic status differences in recreational physical activity levels and real and perceived access to a supportive environment. Prev Med. 2002;35:601611.

18. Karusisi N, Bean K, Oppert JM, Pannier B, Chaix B. Multiple dimensions of residential environments, neighborhood experiences, and jogging behavior in the RECORD Study. Prev Med. 2012;55(1):50-55.

19. Hou N, Popkin BM, Jacobs DR, Jr., et al. Longitudinal associations between neighborhood-level street network with walking, bicycling, and jogging: the CARDIA study. Health Place. 2010; 16(6):1206-1215.
20. Sallis JF, Hovell MF, Hofstetter CR, et al. Distance between homes and exercise facilities related to frequency of exercise among San Diego residents. Public Health Rep. 1990;105(2):179-185.

21. McCormack GR, Giles-Corti B, Bulsara M. Correlates of using neighborhood recreational destinations in physically active respondents. J Phys Act Health. 2007;4(1):39-53.

22. McCormack GR, Giles-Corti B, Bulsara $\mathrm{M}$. The relationship between destination proximity, destination mix and physical activity behaviors. Prev Med. 2008;46(1):33-40.

23. Saelens BE, Sallis JF, Black JB, Chen D. Neighborhood-based differences in physical activity: an environment scale evaluation. Am J Public Health. 2003; 93(9):1552-1558.

24. Giles-Corti B, Timperio A, Bull F, Pikora T. Understanding physical activity environmental correlates: increased specificity for ecological models. Exerc Sport Sci Rev. 2005;33(4):175-181.

25. Cao X, Mokhtarian P, Handy S. Examining the impacts of residential self-selection on travel behaviour: a focus on empirical findings. Transp Rev. 2009;29(3):359-395.

26. McCormack GR, Friedenreich C, Sandalack BA, Giles-Corti B, DoyleBaker PK, Shiell A. The relationship between cluster-analysis derived walkability and local recreational and transportation walking among Canadian adults. Health Place. 2012;18:1079-1087.

27. Jack E, McCormack GR. The associations between objectively-determined and self-reported urban form characteristics and neighborhood-based walking in adults. Int $\mathrm{J}$ Behav Nutr Phys Act [Internet]. 2014 Jun 4;11:71. En ligne à : http://dx.doi.org/10.1186/1479-5868 $-11-71$

28. McCormack GR, Friedenreich C, Shiell A, Giles-Corti B, Doyle-Baker PK. Sexand age-specific seasonal variations in physical activity among adults. J Epidemiol Community Health. 2010;64: 1010-1016. 
29. Oliver L, Schuurman N, Hall A. Comparing circular and network buffers to examine the influence of land use on walking for leisure and errands. Int J Health Geogr [Internet]. 2007 Sep 20;6(1):41. En ligne à : http://dx.doi .org/ 10.1186/1476-072X-6-41

30. Sandalack BA, Alaniz Uribe FG, Eshghzadeh Zanjani A, Shiell A, McCormack GR, Doyle-Baker PK. Neighbourhood type and walkshed size. J Urbanism. 2013;6(3):236-255.

31. Bow CJ, Waters N, Faris $P$, et al. Accuracy of city postal code coordinates as a proxy for location of residence. Int J Health Geogr [Internet]. 2004 Mar 18;3(1):5. En ligne à : http:// dx.doi.org/10.1186/1476-072X-3-5

32. Foster S, Knuiman M, Villanueva K, Wood L, Christian H, Giles-Corti B. Does walkable neighbourhood design influence the association between objective crime and walking? Int $\mathrm{J}$ Behav Nutr Phys Act [Internet]. 2014 Jul 26; 11:100. En ligne à : http://dx.doi.org /10.1186/s12966-014-0100-5

33. Villanueva K, Knuiman M, Nathan A, et al. The impact of neighborhood walkability on walking: does it differ across adult life stage and does neighborhood buffer size matter? Health Place. 2014;25:43-6.

34. McCormack GR, Shiell A, Doyle-Baker PK, Friedenreich C, Sandalack B, Giles-Corti B. Testing the reliability of neighborhood-specific measures of physical activity among Canadian adults. J Phys Act Health. 2009;6(3): 367-373.

35. Giles-Corti B, Timperio A, Cutt H, et al. Development of a reliable measure of walking within and outside the local neighborhood: RESIDE's Neighborhood Physical Activity Questionnaire. Prev Med. 2006;42(6):455-459.

36. van Stralen MM, De Vries H, Mudde AN, Bolman C, Lechner L. Determinants of initiation and maintenance of physical activity among older adults: a literature review. Health Psych Rev. 2009;3(2):147-207.

37. Tremblay MS, Warburton DE, Janssen I, et al. New Canadian physical activity guidelines. Appl Physiol Nutr Metab. 2011;36(1):36-46.
38. Sugiyama T, Neuhaus $M$, Cole $R$, Giles-Corti B, Owen N. Destination and route attributes associated with adults' walking: a review. Med Sci Sports Exerc. 2012;44(7):1275-86.

39. Sugiyama T, Cerin E, Owen N, et al. Perceived neighbourhood environmental attributes associated with adults' recreational walking: IPEN Adult study in 12 countries. Health Place. 2014;28: 22-30.

40. Sandalack B, Nicolai A. The Calgary Project: urban form/urban life. Calgary (AB): University of Calgary Press; 2006. 218 p.

41. Berrigan D, Troiano RP. The association between urban form and physical activity in U.S. adults. Am J Prev Med. 2002;23(2 Suppl 1):74-9.

42. McCormack GR, Rock M, Toohey AM, Hignell D. Characteristics of urban parks associated with park use and physical activity: a review of qualitative research. Health Place. 2010;16(4): 712-726.

43. Koohsari MJ, Mavoa S, Villanueva K, et al. Public open space, physical activity, urban design and public health: concepts, methods and research agenda. Health Place. 2015;33:75-82.

44. Kaczynski A, Henderson K. Environmental correlates of physical activity: a review of evidence about parks and recreation. Leisure Sciences. 2007; 29(4):315-354.

45. Kaczynski AT, Potwarka LR, Saelens BE. Association of park size, distance, and features with physical activity in neighborhood parks. Am J Public Health. 2008;98(8):1451-1456.

46. Sugiyama T, Francis J, Middleton NJ, Owen N, Giles-Corti B. Associations between recreational walking and attractiveness, size, and proximity of neighborhood open spaces. Am J Public Health. 2010;100(9):1752-1757.

47. McCormack GR, Rock M, Swanson K, Burton L, Massolo A. Physical activity patterns in urban neighbourhood parks: insights from a multiple case study. BMC Public Health [Internet]. 2014 Sep 17;14:962. En ligne à : http:// dx.doi.org/10.1186/1471-2458-14-962
48. McCormack GR, Shiell A, Giles-Corti $B$, et al. The association between sidewalk length and walking for different purposes in established neighborhoods. Int J Behav Nutr Phys Act [Internet]. 2012 Aug 1;9:92. En ligne à : http://dx.doi.org/10.1186/1479-5868 $-9-92$

49. Gunn LD, Lee Y, Geelhoed E, Shiell A, Giles-Corti B. The cost-effectiveness of installing sidewalks to increase levels of transport-walking and health. Prev Med. 2014;67:322-9.

50. Lim S, Wyker B, Bartley K, Eisenhower D. Measurement error of self-reported physical activity levels in New York City: assessment and correction. Am J Epidemiol. 2015;181(9):648-655.

51. McCormack G, Shiell, A, Doyle-Baker P, Friedenreich C, Giles-Corti B. Gender and age-specific seasonal variations in physical activity among adults. J Epidemiol Community Health. 2010;64(11):1010-1016.

52. Blumberg SJ, Luke JV, Cynamon ML. Telephone coverage and health survey estimates: evaluating the need for concern about wireless substitution. Am J Public Health. 2006;96(5):926-931.

53. Mayne SL, Auchincloss AH, Michael YL. Impact of policy and built environment changes on obesity-related outcomes: a systematic review of naturally occurring experiments. Obes Rev. 2015;16(5):362-375.

54. Wong BY, Faulkner G, Buliung R. GIS measured environmental correlates of active school transport: a systematic review of 14 studies. Int J Behav Nutr Phys Act [Internet]. 2011 May 6;8:39. En ligne à : http://dx.doi.org/10.1186 /1479-5868-8-39

55. Hall KS, McAuley E. Individual, social environmental and physical environmental barriers to achieving 10000 steps per day among older women. Health Educ Res. 2010;25(3):478-488.

56. Learnihan V, Van Niel KP, Giles-Corti B, Knuiman M. Effect of scale on the links between walking and urban design. Geogr Res. 2011;49(2):183-191. 